\title{
Symplectic diffeomorphisms with inverse shadowing
}

Manseob Lee*

\section{"Correspondence:} Imsds@mokwon.ac.kr Department of Mathematics, Mokwon University, Daejeon 302-729, Korea

\section{Springer}

\begin{abstract}
Let $f$ be a symplectic diffeomorphism of a closed $C^{\infty} 2 n$-dimensional Riemannian manifold $M$. In this paper, we prove the equivalence between the following conditions:

(a) $f$ belongs to the $C^{1}$-interior of the set of symplectic diffeomorphisms satisfying the inverse shadowing property with respect to the continuous methods,

(b) $f$ belongs to the $C^{1}$-interior of the set of symplectic diffeomorphisms satisfying the orbital inverse shadowing property with respect to the continuous methods,

(c) $f$ is Anosov.
\end{abstract}

This result extends Bessa and Rocha's result (Appl. Math. Lett. 25:163-165, 2012).

MSC: $37 C 15 ; 37 C 50$

Keywords: topological stability; inverse shadowing; orbital inverse shadowing; Anosov; symplectic diffeomorphism

\section{Introduction}

Let $M$ be a closed $C^{\infty} 2 n$-dimensional manifold with Riemannian structure and endowed with a symplectic form $\omega$, and let $\operatorname{Diff}_{\omega}(M)$ be the set of symplectomorphisms, that is, of diffeomorphisms $f$ defined on $M$ and such that

$$
\omega_{x}\left(v_{1}, v_{2}\right)=\omega_{f(x)}\left(D_{x} f\left(v_{1}\right), D_{x} f\left(v_{2}\right)\right),
$$

for $x \in M$ and $v_{1}, v_{2} \in T_{x} M$. Consider this space endowed with the $C^{1}$ Whitney topology. It is well known that $\operatorname{Diff}_{\omega}(M)$ is a subset of all $C^{1}$ volume-preserving diffeomorphisms. Denote by $d$ the distance on $M$ induced from a Riemannian metric $\|\cdot\|$ on the tangent bundle TM. By the theorem of Darboux [1, Theorem 1.8], there is an atlas $\left\{\varphi_{i}^{j}: U_{i} \rightarrow \mathbb{R}^{2 n}\right\}$, where $U_{i}$ is an open set of $M$ satisfying $\varphi_{i}^{*} \omega_{0}=\omega$ with $\omega_{0}=\sum_{i=0}^{n} d y_{i} \wedge d y_{n+i}$.

The notion of the inverse shadowing property which is a 'dual' notion of the shadowing property. Inverse shadowing property was introduced by Corless and Pilyugin in [2], and the qualitative theory of dynamical systems with the property was developed by various authors (see [2-4]).

Now, we introduce the inverse shadowing property and some results for the inverse shadowing. Let $M^{\mathbb{Z}}$ be the space of all two-sided sequences $\xi=\left\{x_{n}: n \in \mathbb{Z}\right\}$ with elements $x_{n} \in M$, endowed with the product topology. Let $f: M \rightarrow M$ be a symplectic diffeomorphism. For a fixed $\delta>0$, let $\Phi_{f}(\delta)$ denote the set of all $\delta$-pseudo orbits of $f$. A mapping

(c) 2013 Lee; licensee Springer. This is an Open Access article distributed under the terms of the Creative Commons Attribution License (http://creativecommons.org/licenses/by/2.0), which permits unrestricted use, distribution, and reproduction in any medium, provided the original work is properly cited. 
$\varphi: M \rightarrow \Phi_{f}(\delta) \subset M^{\mathbb{Z}}$ is said to be a $\delta$-method for $f$ if $\varphi(x)_{0}=x$, and each $\varphi(x)$ is a $\delta$-pseudo orbit of $f$ through $x$, where $\varphi(x)_{0}$ denotes the 0th component of $\varphi(x)$. For convenience, write $\varphi(x)$ for $\left\{\varphi(x)_{k}\right\}_{k \in \mathbb{Z}}$. The set of all $\delta$-methods for $f$ will be denoted by $\mathcal{T}_{0}(f, \delta)$. Say that $\varphi$ is continuous $\delta$-method for $f$ if $\varphi$ is continuous. The set of all continuous $\delta$-methods for $f$ will be denoted by $\mathcal{T}_{c}(f, \delta)$. If $g: M \rightarrow M$ is a homeomorphism with $d_{0}(f, g)<\delta$ then $g$ induces a continuous $\delta$-method $\varphi_{g}$ for $f$ by defining

$$
\varphi_{g}(x)=\left\{g^{n}(x): n \in \mathbb{Z}\right\}
$$

Let $\mathcal{T}_{h}(f, \delta)$ denote the set of all continuous $\delta$-methods $\varphi_{g}$ for $f$ which are induced by a homeomorphism $g: M \rightarrow M$ with $d_{0}(f, g)<\delta$, where $d_{0}$ is the usual $C^{0}$-metric. Let $\mathcal{T}_{d}(f, \delta)$ denote by the set of all continuous $\delta$-methods $\varphi_{g}$ for $f$, which are induced by $g \in \operatorname{Diff}_{\omega}(M)$ with $d_{1}(f, g)<\delta$. Then clearly we know that

$$
\mathcal{T}_{d}(f) \subset \mathcal{T}_{h}(f) \subset \mathcal{T}_{c}(f) \subset \mathcal{T}_{0}(f),
$$

$\mathcal{T}_{\alpha}(f)=\bigcup_{\delta>0} \mathcal{T}_{\alpha}(f, \delta), \alpha=0, c, h, d$. We say that $f$ has the inverse shadowing property with respect to the class $\mathcal{T}_{\alpha}(f), \alpha=0, c, h, d$, if for any $\epsilon>0$ there exists $\delta>0$ such that for any $\delta$-method $\varphi \in \mathcal{T}_{\alpha}(f, \delta)$, and for a point $x \in M$ there is a point $y \in M$ such that

$$
d\left(f^{k}(x), \varphi_{g}(y)_{k}\right)<\epsilon, \quad k \in \mathbb{Z} .
$$

We say that $f$ has the orbital inverse shadowing property with respect to the class $\mathcal{T}_{\alpha}(f)$, $\alpha=0, c, h, d$, if for any $\epsilon>0$ there exists $\delta>0$ such that for any $\delta$-method $\varphi \in \mathcal{T}_{\alpha}(f, \delta)$, and for a point $x \in M$ there is a point $y \in M$ such that

$$
d_{H}\left(\overline{\mathcal{O}_{f}(x)}, \overline{\mathcal{O}_{\varphi_{g}}(y)}\right)<\epsilon
$$

where $d_{H}$ is the Hausdorff metric, and $\bar{A}$ is the closure of $A$. We denote by $\mathcal{I} \mathcal{S}_{\omega, \alpha}(M)$ the set of symplectic diffeomorphisms on $M$ with the inverse shadowing property with respect to the class $\mathcal{T}_{\alpha}$ and denote by $\mathcal{O} \mathcal{I S}_{\omega, \alpha}(M)$ the set of symplectic diffeomorphisms on $M$ with the orbital inverse shadowing property respect to the class $\mathcal{T}_{\alpha}$, where $\alpha=a, c, h, d$. Let $\operatorname{int} \mathcal{I S}_{\omega, \alpha}(M)$ be the $C^{1}$-interior of the set of symplectic diffeomorphisms on $M$ with the inverse shadowing property respect to the class $\mathcal{T}_{\alpha}$, and let int $\mathcal{O} \mathcal{I S}_{\omega, \alpha}(M)$ be the $C^{1}$-interior of the set of symplectic diffeomorphisms on $M$ with the orbital inverse shadowing property respect to the class $\mathcal{T}_{\alpha}$, where $\alpha=a, c, h, d$. Note that $f$ has the inverse shadowing property if and only if $f^{n}$ has the inverse shadowing property, for all $n \in \mathbb{Z}$. Lee [3] showed that a diffeomorphism belongs to the $C^{1}$-interior of the set of diffeomorphisms having the inverse shadowing property with respect to the $\mathcal{T}_{d}(f)$ if and only if it is structurally stable. Pilyugin [4] proved that a diffeomorphism belongs to the $C^{1}$-interior of the set of $\mathrm{f}$ diffeomorphisms having the inverse shadowing property with respect to the class $\mathcal{T}_{c}(f)$ if and only if it is structurally stable.

The notion of topological stability was introduced by Walters [5], and he showed that every Anosov diffeomorphism is topological stable. In [6], Nitecki proved that if $f$ satisfies both Axiom A and the strong transversality condition, then it is topological stable. We say that $f$ is topological stable if for any $\epsilon>0$, there is $\delta>0$ such that for any $g \in \operatorname{Diff}(M), \delta-C^{0}$ closed to $f$, there is a continuous map $h: M \rightarrow M$ satisfying $h \circ g=f \circ h$ and $d(f(x), x)<\epsilon$ 
for all $x \in M$. Moriyasu [7] showed that the $C^{1}$-interior of the set of all topologically stable diffeomorphisms is characterized as the set of $C^{1}$-structurally stable. Very recently, Bessa and Rocha [8] proved that if a symplectic diffeomorphism belongs to the $C^{1}$-interior of the set of topologically stable, then the diffeomorphism is Anosov.

Remark 1.1 By the definition of the inverse shadowing, we have the following implication: topological stability $\Rightarrow$ inverse shadowing property with respect to the continuous method $\mathcal{T}_{d} \Rightarrow$ orbital inverse shadowing property with respect to the continuous method $\mathcal{T}_{d}$.

From the above remark, we know that our result is a slight generalization of the main theorem in [8]. In this paper, we omit the phrase 'with respect to the class $\mathcal{T}_{d}$ ' for simplicity. So, we say that $f$ has the inverse shadowing property means that $f$ has the inverse shadowing property with respect to the class $\mathcal{T}_{d}$.

We say that $\Lambda$ is hyperbolic if the tangent bundle $T_{\Lambda} M$ has a $D f$-invariant splitting $E^{s} \oplus$ $E^{u}$ and there exist constants $C>0$ and $0<\lambda<1$ such that

$$
\left\|\left.D_{x} f^{n}\right|_{E_{x}^{s}}\right\| \leq C \lambda^{n} \text { and }\left\|\left.D_{x} f^{-n}\right|_{E_{x}^{u}}\right\| \leq C \lambda^{n}
$$

for all $x \in \Lambda$ and $n \geq 0$. If $\Lambda=M$ then $f$ is Anosov. We define the set $\mathcal{F}_{\omega}(M)$ as the set of diffeomorphisms $f \in \operatorname{Diff}_{\omega}(M)$ which have a $C^{1}$-neighborhood $\mathcal{U}(f) \subset \operatorname{Diff}_{\omega}(M)$ such that if for any $g \in \mathcal{U}(f)$, every periodic point of $g$ is hyperbolic. Then we can see the following.

Lemma 1.2 [6] If $\in \in \mathcal{F}_{\omega}(M)$, then $f$ is Anosov.

Note that $\mathcal{F}_{\omega}(M) \subset \mathcal{F}(M)$ (see [9, Corollary 1.2]). By a result of Newhouse [10], if the symplectic diffeomorphisms is not Anosov then 1-elliptic points can be created by an arbitrary small $C^{1}$-perturbations of the symplectic diffeomorphism.

In this paper, we investigate the cases when a symplectic diffeomorphism $f$ is in $C^{1}$ interior inverse shadowing property with respect to the class $\mathcal{T}_{d}(f)$, then it is Anosov. Let $\operatorname{int} \mathcal{I S}_{\omega}(M)$ be denoted the set of symplectic diffeomorphisms in $\operatorname{Diff}_{\omega}(M)$ satisfying the inverse shadowing property with respect to the class $\mathcal{T}_{d}$, and let int $\mathcal{O} \mathcal{I S}_{\omega}(M)$ be denoted the set of symplectic diffeomorphisms in $\operatorname{Diff}_{\omega}(M)$ satisfying the orbital inverse shadowing property with respect to the class $\mathcal{T}_{d}$ when we mention the inverse shadowing property (resp. orbital inverse shadowing property); that is, the inverse shadowing property (resp. orbital inverse shadowing property)' implies the 'inverse shadowing property (resp. orbital inverse shadowing property) with respect to the class $\mathcal{T}_{d}$. Now we are in position to state the theorem of our paper.

Theorem 1.3 Let $f \in \operatorname{Diff}_{\omega}(M)$. Then

$$
\operatorname{int} \mathcal{I S}_{\omega}(M)=\operatorname{int} \mathcal{O I S}_{\omega}(M)=\mathcal{A N}_{\omega}(M)
$$

where $\mathcal{A N}_{\omega}(M)$ is the set of Anosov symplectic diffeomorphisms in $\operatorname{Diff}_{\omega}(M)$.

\section{Proof of Theorem 1.3}

Let $M$ be as before, and let $f \in \operatorname{Diff}_{\omega}(M)$. Then the following is symplectic version of Franks' lemma. 
Lemma 2.1 [11, Lemma 5.1] Let $f \in \operatorname{Diff}_{\omega}(M)$ and $\mathcal{U}(f)$ be given. Then there are $\delta_{0}>0$ and $\mathcal{U}_{0}(f) \subset \mathcal{U}(f)$ such that for any $g \in \mathcal{U}_{0}(f)$, a finite set $\left\{x_{1}, x_{2}, \ldots, x_{n}\right\}$, a neighborhood $U$ of $\left\{x_{1}, x_{2}, \ldots, x_{n}\right\}$ and symplectic maps $L_{i}: T_{x_{i}} M \rightarrow T_{g\left(x_{i}\right)} M$ satisfying $\left\|L_{i}-D g\left(x_{i}\right)\right\|<\delta_{0}$ for all $1 \leq i \leq n$, there are $\epsilon_{0}>0$ and $\tilde{g} \in \mathcal{U}(f)$ such that

(a) $\tilde{g}(x)=g(x)$ if $x \in M \backslash U$,

(b) $\tilde{g}(x)=\varphi_{g\left(x_{i}\right)} \circ L_{i} \circ \varphi_{x_{i}}^{-1}(x)$ if $x \in B_{\epsilon_{0}}\left(x_{i}\right)$,

where $B_{\epsilon_{0}}\left(x_{i}\right)$ is the $\epsilon_{0}$-neighborhood of $x_{i}$.

A periodic point for $f$ is a point $p \in M$ such that $f^{\pi(p)}(p)=p$, where $\pi(p)$ is the minimum period of $p$. We say that a periodic point $p$ is elliptic if $D_{p} f^{\pi(p)}$ has one nonreal eigenvalue of norm one. We say that a periodic point $p$ is a $k$-elliptic periodic point if for a periodic point $p$ of period $\pi(p)$ the tangent map $D_{p} f^{\pi(p)}$ has exactly $2 k$ simple nonreal eigenvalues of norm 1 and the other ones have norm different from 1 . In dimension 2, then 1-elliptic periodic points are actually elliptic. We say that $p$ is hyperbolic if $D f^{\pi(p)}$ has no norm one eigenvalue. The following facts are enough to prove Theorem 1.3 by Lemma 1.2.

Lemma 2.2 If $f \in \operatorname{Diff}_{\omega}(M)$ has the orbital inverse shadowing property, then $f$ is not the identity map.

Proof Suppose, by contradiction, that $f$ is the identity map. Take $\epsilon=1 / 4$, and let $0<\delta<\epsilon$ be the number of the orbital inverse shadowing property of $f$. Since $f$ is the identity map, we defined $g \in \operatorname{Diff}_{\omega}(M)$ by $g(x)=\left(x_{1}+\delta / 2, x_{2}, \ldots, x_{2 n}\right)$, for $x=\left(x_{1}, x_{2}, \ldots, x_{2 n}\right) \in M$. Then $g \in \mathcal{T}_{d}(f)$. Since $f$ has the orbital inverse shadowing property, there is $y \in M$ such that for any $x \in M$,

$$
d(x, y)<\epsilon \quad \text { and } \quad d_{H}\left(\overline{\mathcal{O}_{f}(y)}, \overline{\mathcal{O}_{g}(x)}\right)<\epsilon
$$

Since $f$ is the identity map and $g$ is an increasing map, there is $k \in \mathbb{N}$ such that $d\left(y, g^{k}(x)\right)>\epsilon$. Thus, by the definition of the Hausdorff metric,

$$
d_{H}\left(\overline{\mathcal{O}_{f}(y)}, \overline{\mathcal{O}_{g}(x)}\right)>\epsilon
$$

This is a contradiction.

Lemma 2.3 If $\in \operatorname{int} \mathcal{I} \mathcal{S}_{\omega}(M)$, then every periodic point off is hyperbolic.

Proof Let $f \in \operatorname{int} \mathcal{I} \mathcal{S}_{\omega}(M)$, and let $\mathcal{U}_{0}(f)$ be a $C^{1}$-neighborhood of $f$. Suppose that there is a $g \in \mathcal{U}_{0}(f)$ such that $g$ have a periodic elliptic point $p$. To simplify, we may assume that $g(p)=p$. Then $D_{p} g$ has $n$ pairs of nonreal eigenvalues, that is, $\left|a_{i}\right|=\left|\bar{a}_{i}\right|=1, i=1, \ldots, n$ with $T_{p} M=E_{p}^{c_{i}} \oplus \cdots \oplus E_{p}^{c_{n}}$ and $\operatorname{dim} E_{p}^{c_{i}}=2, i=1, \ldots, n$. By Lemma 2.1, there are $\alpha>0$ and $g_{1} \in \mathcal{U}(f)$ such that

$$
g_{1}(x)= \begin{cases}\varphi_{g(p)} \circ D_{p} g \circ \varphi_{p}^{-1}(x) & \text { if } x \in B_{\alpha}(p), \\ g(x) & \text { if } x \notin B_{4 \alpha}(p) .\end{cases}
$$

Then $g(p)=g_{1}(p)=p$. 
First, we consider the case $E_{p}^{c_{1}}(\alpha)$ other case is similar. Since $p$ is nonhyperbolic for $g_{1}$, by our construction, we may assume that there is $l>0$ such that $D_{p} g_{1}^{l}(v)=v$ for any $v \in$ $E_{p}^{c_{1}}(\alpha) \cap \varphi_{p}^{-1}\left(B_{\alpha}(p)\right)$. Take $v \in E_{p}^{c_{1}}(\alpha)$ such that $\|v\|=\alpha / 4$. Then we can find a small arc $\mathcal{I}_{p}=\varphi_{p}(\{t v:-\alpha / 4 \leq t \leq \alpha / 4\}) \subset B_{\alpha}(p)$ such that

(i) $g_{1}^{i}\left(\mathcal{I}_{p}\right) \cap g_{1}^{j}\left(\mathcal{I}_{p}\right)=\emptyset$ if $0 \leq i \neq j \leq l-1$, and

(ii) $g_{1}^{l}\left(\mathcal{I}_{p}\right)=\mathcal{I}_{p}$, that is, $\left.g_{1}^{l}\right|_{\mathcal{I}_{p}}$ is the identity map.

Then we can choose $0<\eta<\alpha / 4$ sufficiently small such that $B_{\eta}\left(g_{1}^{i}\left(\mathcal{I}_{p}\right)\right) \cap B_{\eta}\left(g_{1}^{j}\left(\mathcal{I}_{p}\right)\right)=\emptyset$ for all $1 \leq i \neq j \leq l-1$. Take $\epsilon=\eta / 4$, and let $0<\delta<\epsilon$ be the number of the definition of the inverse shadowing property of $g_{1}$ for $\epsilon$. For the $\delta>0$, we can define $\mathcal{T}_{d}\left(g_{1}\right)$-method as follows: Let $\psi \in \operatorname{Diff}_{\omega}(M)$ be such that $p$ is a hyperbolic periodic point for $\psi$ with $\psi(p)=p$ and $d\left(g_{1}, \psi\right)<\delta$. Then $\psi \in \mathcal{T}_{d}\left(g_{1}\right)$. To simplicity, we may assume that $g_{1}^{l}=g_{1}$. Take $y \in B_{4 \epsilon}(p) \cap \mathcal{I}_{p}$ such that $d(y, p)=2 \epsilon$, and $g_{1}^{n}(y)=y$ for all $n \in \mathbb{Z}$. Since $g_{1}$ has the inverse shadowing property, we can see that for any $z \in M$

$$
d\left(g_{1}^{n}(y), \varphi_{\psi}(z)_{n}\right)=d\left(g_{1}^{n}(y), \psi^{n}(z)\right)<\epsilon
$$

for all $n \in \mathbb{Z}$. For any $z \in B_{\epsilon}(p)$, if $z=p$, then since $\left.g_{1}\right|_{\mathcal{I}_{p}}$ is the identity map, it is clear that $g_{1}$ does not have the inverse shadowing property. If $z \neq p$, then since $p$ is a hyperbolic periodic point for $\psi$, there is $k \in \mathbb{Z}$ such that

$$
d\left(g_{1}^{k}(y), \psi^{k}(z)\right)=d\left(y, \psi^{k}(z)\right)>\epsilon
$$

This is a contradiction.

Finally, we may assume that there are $m_{i}$ (the minimum numbers) such that $D_{p} g_{1}^{m_{i}}(v)=v$ for any $v \in E_{p}^{c_{i}}(\alpha) \cap \varphi_{p}^{-1}\left(B_{\alpha}(p)\right), i=2, \ldots, n$. Let $K=\operatorname{lcm}\left\{m_{i}: i=2, \ldots, n\right\}$. Here, lcm is the lowest common multiple.

To simplify, we assume that $g_{2}=g_{1}^{K}$. Since $D_{p} g_{1}^{m_{i}}(v)=v$ for any $v \in E_{p}^{c_{i}}(\alpha) \cap \varphi_{p}^{-1}\left(B_{\alpha}(p)\right)$, $i=2, \ldots, n$, by the above argument, there exists a small $\operatorname{arc} \mathcal{I}_{p} \subset B_{\alpha}(p)$ such that $\left.g_{2}^{l}\right|_{\mathcal{I}_{p}}$ is the identity map, for some $l>0$. Then we can find $\psi \in \mathcal{T}_{d}\left(g_{2}\right)$ such that $p$ is a hyperbolic periodic point for $\psi$ with $\psi(p)=p, d\left(g_{2}, \psi\right)<\delta$. By the inverse shadowing property for $g_{2}$, there exists $y \in B_{4 \epsilon}(p) \cap \mathcal{I}_{p}$ such that

$$
d(y, p)=2 \epsilon \quad \text { and } \quad g_{2}^{l n}(y)=y
$$

for all $n \in \mathbb{Z}$. Then there exists $j \in \mathbb{Z}$ such that

$$
d\left(g_{2}^{j}(y), \psi^{j}(z)\right)=d\left(y, \psi^{j}(z)\right)>\epsilon .
$$

This is a contradiction. Thus, every periodic point of $f \in \operatorname{int} \mathcal{I} \mathcal{S}_{\omega}(M)$ is hyperbolic.

Lemma 2.4 If $f \in \operatorname{int} \mathcal{O} \mathcal{I S}_{\omega}(M)$, then every periodic point off is hyperbolic.

Proof Let $f \in \operatorname{int} \mathcal{O} \mathcal{I S}_{\omega}(M)$. Then as in the proof of Lemma 2.3 and Lemma 2.2, we can obtain a contradiction.

Proof of Theorem 1.3 Let $f \in \operatorname{int} \mathcal{I} \mathcal{S}_{\omega}(M)$, and let $f \in \operatorname{int} \mathcal{O} \mathcal{I} \mathcal{S}_{\omega}(M)$. Suppose that $f \notin$ $\mathcal{F}_{\omega}(M)$. Then there is $g \in \mathcal{U}_{0}(f) \subset \mathcal{U}(f)$ such that $g$ have a periodic elliptic point $p$. By 
Lemma 2.3 and Lemma 2.4, $g$ does not have a periodic elliptic point. This is a contradiction. Thus, if $f \in \operatorname{int} \mathcal{I S}_{\omega}(M)$ or $f \in \operatorname{int} \mathcal{O I S}_{\omega}(M)$ then $f \in \mathcal{F}_{\omega}(M)$. By Lemma $1.2, f$ is Anosov.

\section{Competing interests}

The author declares that they have no competing interests.

\section{Acknowledgements}

We wish to thank the referee for carefully reading the manuscript and providing us many good suggestions. This work is supported by Basic Science Research Program through the National Research Foundation of Korea (NRF) funded by the Ministry of Education, Science and Technology, Korea (No. 2011-0007649).

Received: 5 January 2013 Accepted: 2 April 2013 Published: 16 April 2013

\section{References}

1. Moser, J, Zehnder, E: Notes on Dynamical Systems. Courant Lecture Notes in Mathmatics, vol. 12. Am. Math. Soc., Providence (2005) (New York University, Courant Institute of Mathematical Sciences, New York)

2. Corless, R, Pilyugin, S: Approximate and real trajectories for generic dynamical systems. J. Math. Anal. Appl. 189, 409-423 (1995)

3. Lee, K: Continuous inverse shadowing and hyperbolicity. Bull. Aust. Math. Soc. 67, 15-26 (2003)

4. Pilyugin, S: Inverse shadowing by continuous methods. Discrete Contin. Dyn. Syst. 8, 29-38 (2002)

5. Walters, P: Anosov diffeomorphisms are topological stable. Topology 9, 71-78 (1970)

6. Nitecki, Z: On semi-stability for diffeomorphisms. Invent. Math. 14, 83-122 (1971)

7. Moriyasu, K: The topological stability of diffeomorphisms. Nagoya Math. J. 123, 91-102 (1991)

8. Bessa, M, Rocha, J: A remark on the topological stability of symplectomorphisms. Appl. Math. Lett. 25, 163-165 (2012)

9. Arbieto, A, Catalan, T: Hyperbolicity in the volume preserving scenario. Ergod. Theory Dyn. Syst. (to appear). doi:10.1017/etds.2012.111

10. Newhouse, S: Quasi-elliptic periodic points in conservative dynamical systems. Am. J. Math. 99, 1061-1087 (1975)

11. Horita, V, Tahzibi, A: Partial hyperbolicity for symplectic diffeomorphisms. Ann. Inst. Henri Poincaré, Anal. Non Linéaire 23, 641-661 (2006)

doi:10.1186/1029-242X-2013-174

Cite this article as: Lee: Symplectic diffeomorphisms with inverse shadowing. Journal of Inequalities and Applications 2013 2013:174.

\section{Submit your manuscript to a SpringerOpen ${ }^{\ominus}$ journal and benefit from:}

- Convenient online submission

Rigorous peer review

- Immediate publication on acceptance

- Open access: articles freely available online

- High visibility within the field

- Retaining the copyright to your article 